\title{
Shocks and slip systems: Predictions from a mesoscale theory of continuum dislocation dynamics
}

\author{
S. Limkumnerd ${ }^{\mathrm{a}, *}$, J.P. Sethna ${ }^{\mathrm{b}}$ \\ ${ }^{\mathrm{a}}$ Zernike Institute for Advanced Materials, University of Groningen, 9747 AG, Groningen, The Netherlands \\ ${ }^{\mathrm{b}}$ Laboratory of Atomic and Solid State Physics, Clark Hall, Cornell University, Ithaca, NY 14853-2501, USA
}

Received 4 February 2007; received in revised form 18 July 2007; accepted 26 August 2007

\begin{abstract}
Exploring a recently developed mesoscale continuum theory of dislocation dynamics, we derive three predictions about plasticity and grain boundary formation in crystals. (1) There is a residual stress jump across grain boundaries and plasticity-induced cell walls as they form, which self-consistently acts to attract neighboring dislocations; residual stress in this theory appears as a remnant of the driving force behind wall formation under both polygonization and plastic deformation. We derive the predicted asymptotic late-time dynamics of the grain-boundary formation process. (2) During grain boundary formation at high temperatures, there is a predicted cusp in the elastic energy density. (3) In early stages of plasticity, when only one type of dislocation is active (single-slip), cell walls do not form in the theory; instead we predict the formation of a hitherto unrecognized jump singularity in the dislocation density.
\end{abstract}

(C) 2007 Elsevier Ltd. All rights reserved.

PACS: 46; 91.60.Dc; 91.60.Ed; 47.40.-x

Keywords: Dislocations; Shocks; Burgers equations; Singularity formation; Plasticity

\section{Introduction}

Dislocations in crystals evolve to form structures, especially walls: grain boundaries at high temperatures where climb is allowed, cell boundaries under low-temperature plastic deformation when climb is forbidden. We examine here the detailed predictions of a new variant of continuum dislocation dynamics (Roy and Acharya, 2005; Limkumnerd and Sethna, 2006), that spontaneously forms sharp walls as shock-wave solutions of the partial differential equation (Limkumnerd and Sethna, 2006; Cho, 2006).

How does this new approach fit in to the extensive existing literature on continuum plasticity and dislocation structure formation? First, there are many large-scale simulation studies using discrete dislocations (Holt, 1970; Lepinoux and Kubin, 1987; Gullouglu et al., 1989; Ghoniem et al., 1990; Lubarda et al., 1993; Barts and Carlsson, 1997; Koslowski and Ortiz, 2004; Ramasubramaniam et al., 2007). The computational

*Corresponding author. Tel.: + 31 503638044; fax: + 31503634886 .

E-mail address: s.limkumnerd@rug.nl (S. Limkumnerd).

URL: http://www.lassp.cornell.edu/sethna/sethna.html (J.P. Sethna). 
burden of evolving an enormous number of dislocation segments with long-range interactions is a primary motivation for developing a continuum theory, which replaces the explicit dislocation lines with an average tensorial density. Whatever continuum theory succeeds in describing the collective behavior, these discrete dislocation simulations will remain as the validation underpinning the continuum theory and likely also as its connection to specific materials properties.

Second, there are theories (along with an earlier, scalar attempt (Sethna et al., 2004) to explain wall formation) which ignore the tensor structure of the dislocation density. (a) Macroscopic continuum plasticity often makes use of the simple von Mises law, which presumes an elastic response when a yield stress is reached, after which the distortion tensor evolves according to the local deviatoric stress (stress with the isotropic pressure removed). Generalizations of the von Mises approach which incorporate corrections due to gradients in the local distortion tensor have grown out of early work on size-dependent hardness (Fleck and Hutchinson, 1993) and dislocation patterns (Aifantis, 1984a, b; Walgraef and Aifantis, 1985; Pontes et al., 2005). (b) Models of plasticity in glasses (a somewhat different physical system) describe localized rearrangements of atoms (Falk and Langer, 1998; Langer, 1998), and appear to generate fractal avalanches and crackling noise (Bailey et al., 2007) reminiscent of crackling noise recently observed in crystal plasticity (Zaiser, 2006). (c) There are a variety of reaction-diffusion models which have been used to describe the widths of persistent slip bands and other dislocation patterns (Aifantis, 1984a, b; Walgraef and Aifantis, 1985; Pontes et al., 2005), cellular structures (Kratochvil, 1990a, b), double cross-slip (Bréchet and Louchet, 1988), dislocation vein structures (Saxlová et al., 1997), and many other effects (Hähner, 1996). In the domains for which these theories were developed, the dislocation density or its tensor structure can be argued to be largely irrelevant; for example, the isotropic theory of work hardening is a reasonable first approximation to macroscopic plasticity. But by omitting explicit evolution of the dislocation density tensor, these approaches lose the ability to predict the rotational and deformation morphology of the mesoscopic dislocation structures, and they lose the connection between the microscopic Peach-Koehler forces on the dislocations and the resulting continuum dynamics - both crucial properties that we want to incorporate into our theory.

Third, there are theories which incorporate more microscopic detail about the dislocation content than we keep, keeping track not only of the net dislocation density but of local dislocation densities for each slip system (including oppositely oriented Burger's vectors), and incorporating dislocation entanglement as effective hardening rules coupling the densities on different slip systems. These researchers have studied both texture (grain orientation distribution) evolution in polycrystal plasticity and the evolution of subgrain structures, either for their own sake (Mika and Dawson, 1999; Barton and Dawson, 2001; Dawson et al., 2002; Arsenlis and Parks, 2002; Arsenlis et al., 2004; Ma et al., 2006) or as a precursor for other computations (like recrystallization simulations (Raabe and Becker, 2000)). Of these three features missing in our model (canceling 'geometrically unnecessary' dislocations, entanglement, and slip systems) the first can plausibly be ignored on the mesoscale. In macroscopic plasticity, most dislocations cancel out in the net density; ignoring the geometrically unneccessary dislocations would be a poor description. On the mesoscale, the crystal misorientations across cell walls and grain boundaries, and the accepted microscopic structure of grain boundaries (Hirth and Lothe, 1992), are solely due to the net dislocation density kept in our model. (Indeed, the geometrically unnecessary dislocations on the macroscale could plausibly be largely due to cancellations between dislocations on mesoscopically separated walls.) The other two features we omit clearly remain important on the mesoscale. Indeed, in the simulations presented here the dislocations evolve in time, where in practice they evolve under increasing strain - a reflection of the lack of entanglement or work hardening in our model. However, our mesoscale theory, by ignoring entanglement and slip planes, does succeed in providing a striking explanation for wall formation that has not emerged analytically from these more detailed theories (although wall formation may have been observed numerically in these models (Mika and Dawson, 1999; Barton and Dawson, 2001; Dawson et al., 2002)). The more detailed and quantitative predictions of the theory presented here must be interpreted as a first approximation, to which the effects of entanglement, slip planes, anisotropy, and materials properties will need to be added.

Finally, Ortiz and collaborators have extended the mathematical minimizing-sequence techniques developed for studying martensitic and magnetic microstructures to describe the formation of dislocation microstructures (Ortiz and Repetto, 1999; Ortiz et al., 2000; Aubry and Ortiz, 2003; Conti and Ortiz, 2005). While it is perhaps too early to draw broad generalizations, it would seem that the model presented here 
quantifies the formation of the dislocation walls, and provides a microscopic model for studying their morphology and evolution, whereas the variational methods directly solve for the microstructure and not how it originates.

The model we study was originally proposed by Roy and Acharya (2005), who allowed both glide and climb. We subsequently rediscovered this law from the microscopic dynamics and a closure approximation, proposed a modified law to suppress climb, and showed numerically that the models developed shock singularities (Limkumnerd and Sethna, 2006), providing a potential underlying explanation for both the grain boundaries formed at high temperatures and the cell walls formed at low temperatures in plastically deformed crystals. In Section 2 we will present Roy and Acharya's derivation for the equations of motion.

In Section 3 we will analyze the solution of our model in one dimension. Near a locally-flat dislocation wall, the properties may be approximated by a 1D theory, incorporating variations only perpendicular to the wall; hence the solution in one dimension likely encapsulates the singular form for wall singularity formation in three dimensions. We will map the dislocation dynamics theory in one dimension onto Burgers equation, the prototype of a partial differential equation exhibiting shock formation. We will analyze the asymptotics of the evolution of the stress and dislocation density near the walls, and show how the residual stress associated with the wall draws in and absorbs neighboring dislocations. We shall also show that the continuum model predicts that the energy density will be continuous across the grain boundary (despite the jumps in the individual residual stress components), but that it is predicted to have a cusp singularity.

In Section 4 we examine the behavior of our model under single-slip conditions - well studied numerically with 2D discrete dynamics simulations, and characteristic of early stages of plastic deformation in certain geometries. When appropriately restricted to forbid climb, the model predicts that materials with only one kind of dislocation will not form cell walls, agreeing with the behavior seen in simulations and experiments. However, the mesoscale theory predicts that these systems will form a more subtle, hitherto unobserved jump singularity in the dislocation density.

Finally, in Section 5 we will consider how and whether these predictions are likely to depend upon the detailed structure of the continuum model, and how physical mechanisms ignored by the model will likely affect and modify these striking predictions.

\section{Governing equations}

A complete macroscopic description of the deformation $\mathbf{u}$ of a material is given by $\partial_{i} u_{j}=\beta_{i j}^{\mathrm{E}}+\beta_{i j}^{\mathrm{P}}$, where $\beta_{i j}^{\mathrm{E}}$ represents the elastic, reversible distortion and the plastic distortion tensor $\beta_{i j}^{\mathrm{P}}$ describes the irreversible plastic deformation. The plastic distortion is the result of the net density of dislocations, described by the Nye dislocation density tensor (Nye, 1953; Eshelby, 1956; Kosevich, 1962; Kröner, 1958; Mura, 1963)

$$
\rho_{i j}(\mathbf{x})=-\varepsilon_{i l m} \partial_{l} \beta_{m j}^{\mathrm{P}}=\sum_{\alpha} t_{i}^{\alpha} b_{j}^{\alpha} \delta\left(\xi^{\alpha}\right)
$$

which measures the net flux of dislocation $\alpha$, tangent to $\mathbf{t}$, with Burgers vector $\mathbf{b}$, in the (coarse-grained) neighborhood of $\mathbf{x}$. The microscopic statement that dislocations cannot end implies $\partial_{i} \rho_{i j}=0$, so the time evolution must be given in terms of a current $\boldsymbol{J}$ (Kosevich, 1962; Mura, 1963; Rickman and Vinãls, 1997):

$$
\partial_{t} \rho_{i j}=-\varepsilon_{i l m} \partial_{l} J_{m j}
$$

so $J_{i j}=\partial_{t} \beta_{i j}^{\mathrm{P}}$. The evolution law is thus determined by the current $\boldsymbol{J}$. We controlled the microscopic mobility difference between glide and climb (Limkumnerd and Sethna, 2006) by introducing a mesoscale parameter $\lambda$. By setting

$$
\partial_{t} \beta_{i j}^{\mathrm{P}}=J_{i j}=J_{i j}^{\mathrm{RA}}-\frac{\lambda}{3} \delta_{i j} J_{k k}^{\mathrm{RA}},
$$

at low temperatures $\lambda=1$ removed the trace of $\boldsymbol{J}$ enforcing volume conservation, and hence forbids climb, while at high temperatures $\lambda=0$ allowed for equal mobilities for both glide and climb.

The current from a single dislocation moving with velocity $\mathbf{v}$ is $J_{i j}=\varepsilon_{i l n} t_{l} b_{j} v_{n} \delta(\xi)$, and the net Peach-Koehler force on a dislocation driving its motion is $f_{l}^{\mathrm{PK}}=-\varepsilon_{l m n} t_{m} b_{c} \sigma_{n c}$ where $\boldsymbol{\sigma}$ is the local stress 
(due, for example, to the other dislocations). In our derivation of the equation of motion (Limkumnerd and Sethna, 2006), we allowed each dislocation to move independently, and then made a closure approximation to write $\boldsymbol{J}$ in terms of $\boldsymbol{\rho}$. Roy and Acharya (2005) got the same final result by simply assuming that all the dislocations move with the same velocity $\mathbf{v}$, given by $D(\rho) / 2$ times the force density $\mathscr{F}_{l}=-\varepsilon_{l m n} \rho_{m c} \sigma_{n c}$ on the local dislocations

$$
J_{i j}^{\mathrm{RA}}=\frac{D}{2} \varepsilon_{i a l} \mathscr{F}_{l} \rho_{a j}=-\frac{D}{2} \varepsilon_{i a l}\left(\varepsilon_{l m n} \rho_{m c} \sigma_{n c}\right) \rho_{a j}=\frac{D}{2}\left(\sigma_{i c} \rho_{a c}-\sigma_{a c} \rho_{i c}\right) \rho_{a j} .
$$

A physically natural choice for $D(\rho)$ is proportional to an inverse density of dislocation lines (so that the force per dislocation drives the motion Roy and Acharya, 2005). Groma et al. (Groma, 1997; Zaiser et al., 2001) use a similar approach to study plastic deformation in two dimensions with only one slip system (i.e., allowing only parallel edge dislocations with one direction of Burgers vector, leading to scalar order parameters). They do a closure-like factorization of a two-point dislocation density correlation function which leads to a theory with one fewer factor of $\rho$ in the evolution law than our equation has. While we cannot generalize their approach to the 3D tensor theory, we can reproduce their continuum theory by choosing $D(\boldsymbol{\rho})=D_{0} / \sqrt{\operatorname{tr}\left(\boldsymbol{\rho}^{\dagger} \boldsymbol{\rho}\right)}$ and specializing to two dimensions and one slip system. We choose here to discuss the mathematically more convenient choice of a constant $D$ (allowing us in Section 3 to map the dynamics onto Burgers equation). Numerically, both choices give qualitatively similar evolution (Limkumnerd and Sethna, 2006).

\section{Analysis of the continuum equation in one dimension}

Near a wall singularity (say, perpendicular to $\hat{\mathbf{z}}$ ) the dynamics is $1 \mathrm{D}$. The variations of the stress, plastic strain, and dislocation densities parallel to the wall asymptotically become unimportant compared to the variations along $\hat{\mathbf{z}}$ as one approaches the singularity. In one dimension the stress $\boldsymbol{\sigma}(\mathbf{x})$, generally given by a long-range integral over the neighboring dislocations $\boldsymbol{\rho}\left(\mathbf{x}^{\prime}\right)$, can be written as a linear function of the local plastic distortion $\boldsymbol{\beta}^{\mathrm{P}}$. In general, the stress field generated by a dislocation density $\boldsymbol{\rho}$ is given in Fourier space by Hirth and Lothe (1992),

$$
\widetilde{\sigma}_{\alpha \beta}(\mathbf{k})=K_{\alpha \beta \mu v}(\mathbf{k}) \widetilde{\rho}_{\mu v}(\mathbf{k}),
$$

where

$$
K_{\alpha \beta \mu v}(\mathbf{k})=-\frac{i \mu k_{\gamma}}{k^{2}}\left[\varepsilon_{\gamma v \alpha} \delta_{\beta \mu}+\varepsilon_{\gamma \nu \beta} \delta_{\alpha \mu}+\frac{2 \varepsilon_{\gamma \nu \mu}}{1-v}\left(\frac{k_{\alpha} k_{\beta}}{k^{2}}-\delta_{\alpha \beta}\right)\right] .
$$

By substituting this form of $\widetilde{\boldsymbol{\rho}}=\widetilde{\boldsymbol{\rho}}\left(k_{z}\right) \delta\left(k_{x}\right) \delta\left(k_{y}\right)$ in one dimension into Eq. (5) together with Eq. (1), one obtains, in real space,

$$
\sigma_{i j}=-\bar{C}_{i j k m} \beta_{k m}^{\mathrm{P}}
$$

where

$$
\bar{C}_{i j k m}=\mu\left(\bar{\delta}_{i k} \bar{\delta}_{j m}+\bar{\delta}_{i m} \bar{\delta}_{j k}+\frac{2 v}{1-v} \bar{\delta}_{i j} \bar{\delta}_{k m}\right),
$$

(a form slightly different from the elasticity tensor), and the Kronecker delta is modified such that $\bar{\delta}_{z z}=0$. The elastic energy density $\mathscr{E}$ in one dimension can (surprisingly) be written in terms of the local plastic distortion tensor

$$
\begin{aligned}
\mathscr{E} & =\frac{1}{2} \bar{C}_{i j k m} \beta_{i j}^{\mathrm{P}} \beta_{k m}^{\mathrm{P}} \\
& =\frac{\mu}{2}\left(\beta_{x y}^{\mathrm{P}}+\beta_{y x}^{\mathrm{P}}\right)^{2}+\mu\left(\beta_{x x}^{\mathrm{P}}{ }^{2}+\beta_{y y}^{\mathrm{P}}{ }^{2}\right)+\frac{\mu \nu}{1-v}\left(\beta_{x x}^{\mathrm{P}}+\beta_{y y}^{\mathrm{P}}\right)^{2},
\end{aligned}
$$

as can be verified by direct computation. Our equation of motion for the plastic distortion $\boldsymbol{\beta}^{\mathrm{P}}$ also simplifies dramatically. The $\beta_{z j}^{\mathrm{P}}$ components do not evolve in one dimension (except $\beta_{z z}^{\mathrm{P}}$ for $\lambda \neq 0$, which helps enforce volume conservation); we can see this from Eq. (4), where $\sigma_{z c}=0$ (because $\bar{C}_{z c k m}=0$ in Eq. (8)) and $\rho_{z c}=0$ (because $\beta$ depends only on $z$ in Eq. (1)). (This equation also shows that the $\beta_{z j}^{\mathrm{P}}$ components are compatible; 
variations in them do not generate dislocation density.) All other components of $\boldsymbol{\beta}^{\mathrm{P}}$ evolve according to

$$
\partial_{t} \beta_{i j}^{\mathrm{P}}=-\frac{1}{2}\left(\partial_{z} \mathscr{E}\right) \partial_{z}\left(\beta_{i j}^{\mathrm{P}}-\frac{\lambda}{3} \beta_{k k}^{\mathrm{P}} \delta_{i j}\right),
$$

where $\mathscr{E}$ is the elastic energy density from Eq. (10) and we have rescaled the time to set $D=1$ (which can again be verified by direct substitution).

We now specialize to the case of $\lambda=0$, where glide and climb are treated on an equal footing (applicable to grain boundary formation during polygonization at high temperatures, for example). In this case, Eq. (10) tells us that the individual components of $\boldsymbol{\beta}^{\mathrm{P}}$ are all independent of one another, slaves solely to the evolution of the total stress energy density $\mathscr{E}$. By contracting Eq. (10) with $\bar{C}_{i j k m} \beta_{k m}^{\mathrm{P}}$ and then using expression 9, the time evolution of the strain energy becomes

$$
\partial_{t} \mathscr{E}+\frac{1}{2}\left(\partial_{z} \mathscr{E}\right)^{2}=0
$$

Eq. (11) can be cast into the famous Burgers equation by defining $\mathscr{F}=\partial_{z} \mathscr{E}$ (Frisch and Bec, 2001; Sethna et al., 2004; Whitham, 1974):

$$
\partial_{t} \mathscr{F}+\mathscr{F} \partial_{z} \mathscr{F}=0 .
$$

The scalar $\mathscr{F}(z)$ is again the net Peach-Koehler force density on the local dislocation density $\rho(z)$. Burgers equation is the archetype of hyperbolic partial differential equations. Under Burgers equation $\mathscr{F}$ will develop sharp jumps downward after a finite evolved time, corresponding to cusps in the energy density $\mathscr{E}$ and leading to jumps in the components of $\boldsymbol{\beta}^{\mathrm{P}}$ (Fig. 1). In particular, from Eq. (1), a jump in $\beta_{x z}^{\mathrm{P}}$ or $\beta_{y z}^{\mathrm{P}}$ corresponds to a tilt boundary, and a jump in $\beta_{x y}^{\mathrm{P}}-\beta_{y x}^{\mathrm{P}}$ is equivalent to a twist boundary. (Note that $\boldsymbol{\beta}^{\mathrm{P}}$ is not a symmetric tensor.) The jump discontinuities in $\beta_{x x}^{\mathrm{P}}, \beta_{y y}^{\mathrm{P}}$, and $\beta_{x y}^{\mathrm{P}}+\beta_{y x}^{\mathrm{P}}$ produce the necessary stress jumps that drive the formation of walls.

Our theory provides specific, quantitative, testable predictions for the dynamics of wall formation. The singularity formation is generic, happening for almost all initial conditions. At late times, the asymptotic latetime solutions to Burgers equation (between the singularities) are linear functions of $z$ whose slopes decay with time

$$
\mathscr{F} \sim \frac{z-z_{0}}{t-t_{0}} .
$$

The constants $z_{0}$ and $t_{0}$ represent arbitrary constants, defining the location of singularities in space and time. The corresponding elastic energy density (continuous across the singularities) has asymptotic form given by integrating Eq. (13)

$$
\mathscr{E} \sim \frac{1}{2} \frac{\left(z-z_{0}\right)^{2}}{\left(t-t_{0}\right)}+\mathscr{E}_{0}
$$

The individual components of the distortion tensor (apart from the three time-independent components $\beta_{z j}$ ) numerically take the form

$$
\beta_{i j}^{\mathrm{P}} \sim \alpha_{i j} \frac{z-z_{0}}{\sqrt{t-t_{0}}}+\gamma_{i j}
$$

which can be shown to be consistent with the evolution law for the energy density (Eq. (14)), so long as the coefficients $\alpha_{i j}$ and $\gamma_{i j}$ obey the relations

$$
\bar{C}_{i j k m} \alpha_{i j} \alpha_{k m}=1, \quad \bar{C}_{i j k m} \alpha_{i j} \gamma_{k m}=0, \quad \bar{C}_{i j k m} \gamma_{i j} \gamma_{k m}=2 \mathscr{E}_{0}
$$

for $i, j=x$ or $y$; there are no restrictions on $\alpha_{i z}$ and $\gamma_{i z}$.

Our theory predicts that, when $\left|t-t_{0}\right| \rightarrow \infty$, the Peach-Koehler attraction that drives the formation of walls, and hence the residual stress, vanishes. All components of $\beta_{i j}^{\mathrm{P}}$ (except for the three non-evolving components $\beta_{z j}^{\mathrm{P}}$ ) exhibit sharp jumps separated by completely flat regions - corresponding to walls of dislocations separating dislocation-free grains. 


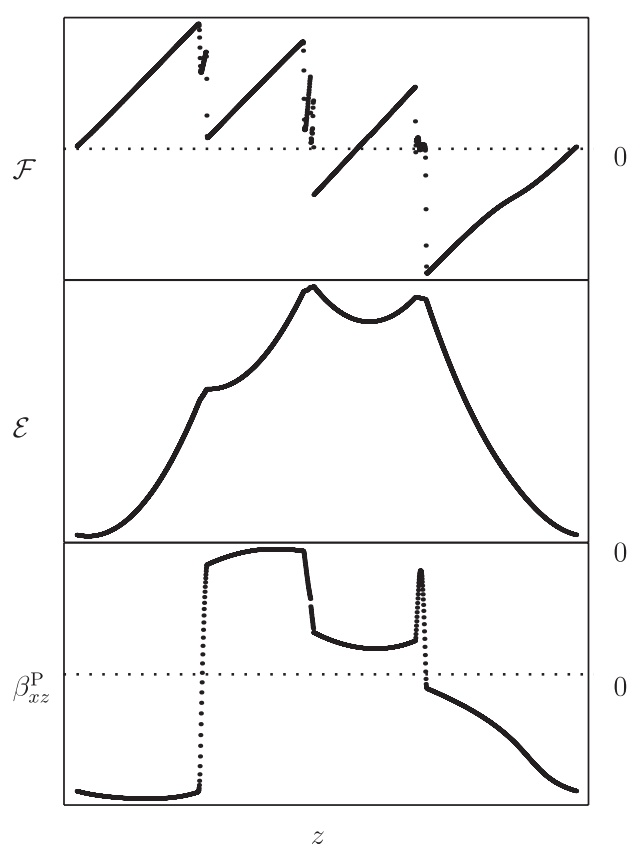

Fig. 1. Cusps and jumps in one dimension. For the continuum dynamics equation allowing both glide and climb, the Peach-Koehler force density $\mathscr{F}$ obeys Burgers equation, and hence develops sharp jumps (top). The individual components of the plastic distortion tensor $\boldsymbol{\beta}^{\mathrm{P}}$ (bottom), as well as the stress and strain tensors, evolve to also have sharp jumps at the walls; the dislocation density hence develops the $\delta$ function singularity associated with grain boundary formation. These discontinuities in the residual stresses and strains, however, cancel out in the net elastic energy density, which is continuous with only a cusp at the walls (middle). All of our simulations have periodic boundary conditions, zero external loading and strain, and are initialized with Gaussian random plastic distortion tensors (whose correlation length governs the initial length scale between walls).

\section{Single-slip: simulations and singularity formation}

There has been extensive work on discrete point dislocation simulations in two dimensions, where it appears necessary to include more than one slip system to form walls (Benzerga et al., 2004; Benzerga etal, 2005; Fournet and Salazar, 1996; Gómez-García et al., 2006; Groma and Bakó, 2000; Groma and Pawley, 1993a, b; Gullouglu and Hartly, 1993), unless dislocation climb motions are allowed in the system (Barts and Carlsson, 1997). What does the continuum model predict for this case? Let us consider a $2 \mathrm{D}$ system (constant along $\hat{\mathbf{z}}$ ) of straight edge dislocations with Burgers vector along $\hat{\mathbf{x}}$, described by a single non-zero component $\rho_{z x}(x, y)$. Such a system has two non-zero components of the distortion tensor, $\rho_{z x}=-\partial_{x} \beta_{y x}^{\mathrm{P}}+\partial_{y} \beta_{x x}^{\mathrm{P}}$. A simulation of this system with Gaussian random initial $\beta_{y x}^{\mathrm{P}}$ and $\beta_{x x}^{\mathrm{P}}$, allowing both glide and climb, generates a series of walls of dislocations roughly parallel to the $\hat{y}$-axis (Fig. 2) similar to those seen by Barts and Carlsson (1997) in their 2D study of single-slip with both glide and climb.

To forbid climb in this case it is convenient ${ }^{1}$ to simply choose $\beta_{x x}^{\mathrm{P}} \equiv 0$. Fig. 3 shows the evolution of the distortion field for climb-free dynamics with a single-slip system. ${ }^{2}$ In agreement with experiment and the discrete dislocation simulations (Bakó and Groma, 1999; Miguel et al., 2001), we observe no cell wall structures in single-slip (which would correspond to jumps in $\boldsymbol{\beta}^{\mathrm{P}}$ in Fig. 3). Instead, we find a network of surfaces exhibiting a striking new singularity: a cusp in the distortion tensor, corresponding to a jump in the dislocation density. We can understand this singularity analytically using our mapping to Burgers equation.

\footnotetext{
${ }^{1}$ Our proposed evolution law Eq. (3) suppressed climb by removing the trace of the current, hence the term $-\lambda / 3 J_{k k} \delta_{i j}$ in $\partial_{t} \beta_{i j}^{\mathrm{P}}$. This choice is inconvenient here, because it introduces new components $\beta_{y y}^{\mathrm{P}}$ and $\beta_{z z}^{\mathrm{P}}$ to the problem, and the corresponding dislocation densities $\rho_{z y}, \rho_{x z}$, and $\rho_{y z}$. While these are allowed by symmetry, they are not part of the discrete dislocation simulation.

${ }^{2}$ Forbidding climb in this $2 \mathrm{D}$ simulation with only one Burgers vector confines the dynamics to one slip system, even though slip systems are not explicitly included into the mesoscale theory.
} 


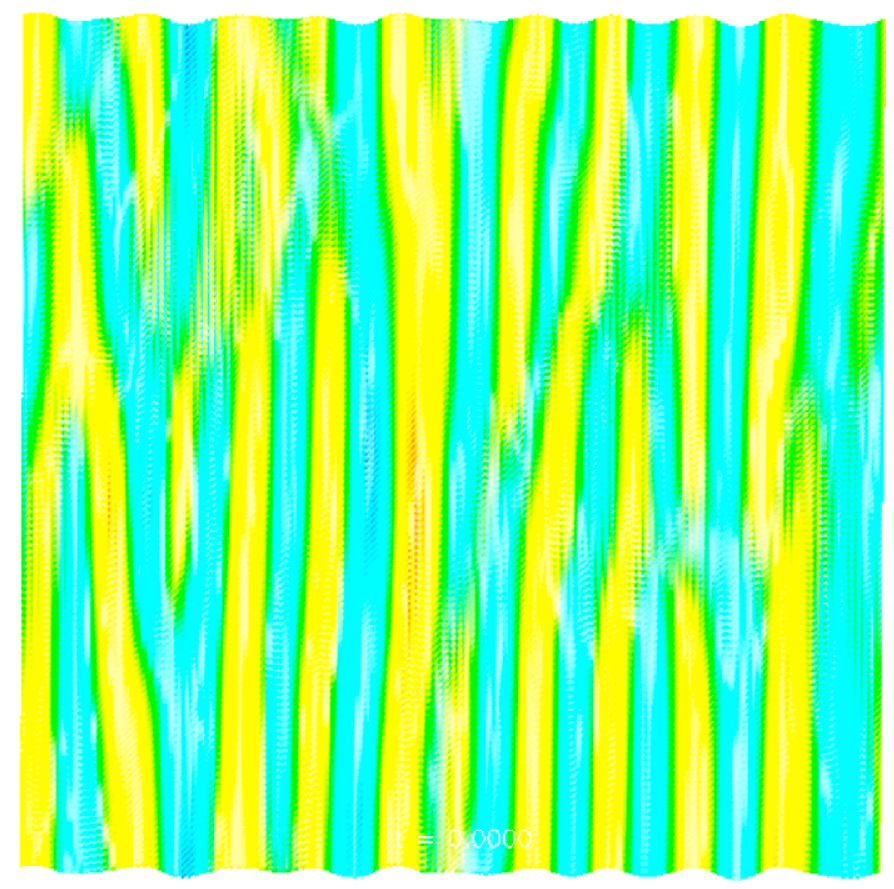

Fig. 2. Continuum of walls. The dislocation density tensor $\rho_{z x}$ evolved allowing both glide and climb from a random initial state of edge dislocations along $\mathbf{t} \sim \hat{\mathbf{z}}$ with $\mathbf{b} \sim \hat{\mathbf{x}}$. Notice that the dislocations arrange themselves into small-angle tilt boundaries at the lattice scale, but do not coarsen; compare to Barts and Carlsson (1997).

With only one non-zero component of $\boldsymbol{\beta}^{\mathrm{P}}$, the energy density is proportional to the square of $\beta_{y x}^{\mathrm{P}}$ (Eq. (9)). The energy density satisfies Eq. (11) and forms cusps, so the distortion tensor must also form cusps.

\section{Discussions}

Which of these new predictions of the mesoscale dislocation dynamics model can be trusted? Which seem (in retrospect) physically plausible, and which are likely artifacts of simplifications made in the modeling process, or just mathematical curiosities of this particular model?

(1) Residual stress. The primary driving force for dislocation motion is stress. In cases where dislocations dynamically assemble into walls (polygonization at high temperatures, cell wall formation at low temperatures), it does seem natural in retrospect to expect that the walls will be associated with stress jumps designed to attract residual dislocations to the walls. Grain boundaries formed from the melt when separate growing crystals touch should likely not be described by this mesoscale continuum model. These residual stress jumps must be viewed as a fundamental prediction of the model. ${ }^{3}$

(2) Cusps in the energy density. If there are jumps in the stress at grain boundaries, surely it is natural that there be some singularity in the energy density. At late stages when the dislocations between grain boundaries have all been removed, a flat boundary can lower the system energy by moving into the region of higher energy density. If both glide and climb are allowed, ${ }^{4}$ and if dislocation mobility is unimpeded (by precipitates, impurities, lattice pinning, or tangling) this traction will lead the boundaries to move until the energy density is continuous across the boundary. Hence, for mobile walls at high temperatures, it is natural to expect the energy density to be continuous, and have only cusp singularities.

(3) Dislocation density jumps in single-slip. The formation of dislocation walls in the continuum theory cannot properly be called a prediction, since wall formation is well-known experimentally. It is, however, a

\footnotetext{
${ }^{3}$ One concern we do have is that a wall with a stress jump can lower its energy by splitting in two; stress jumps at walls in real materials may be stabilized by a different mechanism than in the continuum model.

${ }^{4}$ The glide-only continuum model does have small jumps in the energy density.
} 


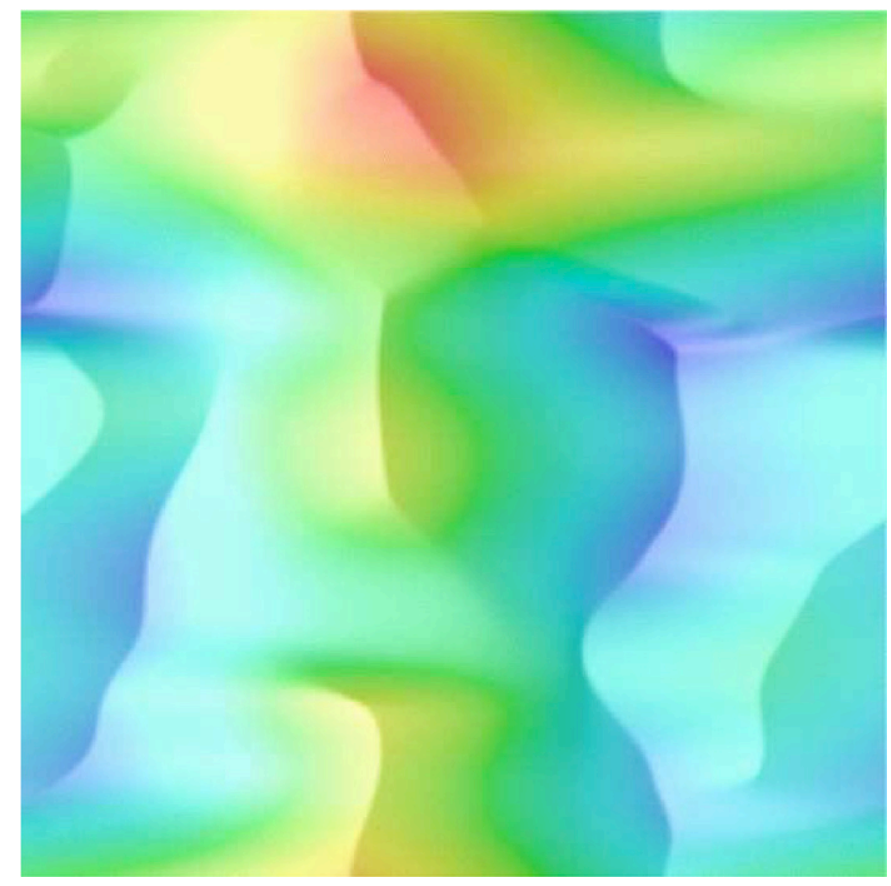

Fig. 3. Cusps formed with one slip system. Plastic distortion tensor $\beta_{y x}^{\mathrm{P}}$ formed by climb-free evolution of a Gaussian random initial state of edge dislocations pointing along $\hat{\mathbf{z}}$ with Burgers vector along $\pm \hat{\mathbf{x}}$. Notice that walls do not form with one slip system, only cusps in the distortion tensor; compare to Miguel et al. (2001).

natural consequence of the hyperbolic form of the equations. The (hitherto unobserved) prediction of dislocation density jumps in early stages of plasticity when only one slip system is active is also a natural consequence of hyperbolic equations. Our continuum model is guaranteed to lower the net energy with time, reassuring us that the predicted dislocation density jumps are energetically favorable and satisfy all compatibility constraints. They could, however, be smeared by pinning and inhomogeneities in real systems just as grain boundaries and cell walls are distorted by these effects. A smeared dislocation density jump may be more challenging to identify than a smeared wall of dislocations, perhaps explaining why these jumps have not yet been seen experimentally.

Are cell walls formed to minimize the energy (the LEDS hypothesis (Kuhlmann-Wilsdorf, 1989, 1995)), or do they form in a non-equilibrium tangling-induced instability? On the one hand, our current model completely lacks effects of dislocation entanglement which induce work hardening. The fact that our walls form from relatively homogeneous initial conditions suggests that a theory evolving to locally minimize the energy does form walls. On the other hand, our theory forms walls to minimize the energy, but they are sharp only because of the particular dynamical evolution law by which they are formed; a continuum blur of walls, within our theory, would also minimize the energy (Limkumnerd and Sethna, 2007).

In summary, we predict that residual stress is not due to inhomogeneities or other infelicities in the formation process, but is an intrinsic component of the formation of grain boundaries and cell walls. We make concrete predictions about the nature and form of these stresses near grain boundaries, that should be testable in colloidal systems or using next-generation X-ray sources. We provide a mesoscale explanation for a key feature of single-slip plasticity (the lack of cell walls), and we predict that these systems will instead form a hitherto unobserved dislocation density jump structure.

\section{Acknowledgments}

We thank Amit Acharya for helpful conversations, and acknowledge funding from NSF Grants ITR/ASP ACI0085969 and DMR-0218475. 


\section{References}

Aifantis, E.C., 1984a. On the microstructural origin of certain inelastic models. Trans. ASME J. Eng. Mater. Technol. 106, 326-330. Aifantis, E.C., 1984b. Towards a continuum approach to dislocation patterning. In: Markenscoff, X. (Ed.), Dislocations in SolidsRecent Advances, AMD-63, ASME, New York, pp. 23-33.

Arsenlis, A., Parks, D.M., 2002. Modeling the evolution of crystallographic dislocation density in crystal plasticity. J. Mech. Phys. Solids 50, 1979-2009.

Arsenlis, A., Parks, D.M., Becker, R., Bulatov, V.V., 2004. On the evolution of crystallographic dislocation density in non-homogeneously deforming crystals. J. Mech. Phys. Solids 52, 1213-1246.

Aubry, S., Ortiz, M., 2003. The mechanics of deformation-induced subgrain-dislocation structures in metallic crystals at large strains. Proc. R. Soc. London A 451, 3131-3158.

Bailey, N.P., Schiøtz, J., Lemaître, A., Jacobsen, K.W., 2007. Avalanche size scaling in sheared three-dimensional amorphous solid. Phys. Rev. Lett. 98, 095501.

Bakó, B., Groma, I., 1999. Stochastic approach for modeling dislocation patterning. Phys. Rev. B 60 (1), $122-127$.

Barton, N.R., Dawson, P.R., 2001. On the spatial arrangement of lattice orientations in hot-rolled multiphase titanium. Modelling Simul. Mater. Sci. Eng. 9, 433-463.

Barts, D.B., Carlsson, A.E., 1997. Simulation and theory of polygonization in single glide. Philos. Mag. A 75, 541-562.

Benzerga, A.A., Bréchet, Y., Needleman, A., Van der Giessen, E., 2004. Incorporating three-dimensional mechanisms into twodimensional dislocation dynamics. Modelling Simul. Mater. Sci. 12, 159-196.

Benzerga, A.A., Bréchet, Y., Needleman, A., Van der Giessen, E., 2005. The stored energy of cold work: predictions from discrete dislocation plasticity. Acta Materialia 53, 4765-4779.

Bréchet, Y., Louchet, F., 1988. Dislocation patterning in uniaxial deformation. Solid State Phenom. 3-4, 335-346.

Cho, A., 2006. Theory of shock waves clears up the puzzling graininess of crystals. Science $311,1361$.

Conti, S., Ortiz, M., 2005. Dislocation microstructures and the effective behavior of single crystals. Arch. Rational Mech. Anal. 176, 103-147.

Dawson, P.R., Mika, D.P., Barton, N.R., 2002. Finite element modeling of lattice misorientations in aluminum polycrystals. Scr. Materialia 47, 713-717.

Eshelby, J.D., 1956. Solid State Physics 3. Academic Press, San Diego.

Falk, M.L., Langer, J.S., 1998. Dynamics of viscoplastic deformation in amorphous solids. Phys. Rev. E 57, 7192-7205.

Fleck, N.A., Hutchinson, J.W., 1993. A phenomenological theory for strain gradient effects in plasticity. J. Mech. Phys. Solids 41, 1825-1857.

Fournet, R., Salazar, J.M., 1996. Formation of dislocation patterns: computer simulations. Phys. Rev. B 53, $6283-6290$.

Frisch, U., Bec, J., 2001. Burgulence. In: Lesieur, M., Yaglom, A., David, F. (Eds.), Les Houches 2000: New Trends in Turbulence. Springer EDP-Sciences, Berlin, pp. 341-383.

Ghoniem, N.M., Matthews, J.R., Amodeo, R.J., 1990. A dislocation model for creep in engineering materials. Res. Mech. 29 , 197-219.

Gómez-García, D., Devincre, B., Kubin, L., 2006. Dislocation patterns and the similitude principle: 2.5d mesoscale simulations. Phys. Rev. Lett. 96, 125503.

Groma, I., 1997. Link between the microscopic and mesoscopic length-scale description of the collective behavior of dislocations. Phys. Rev. B 56 (10), 5807-5813.

Groma, I., Bakó, B., 2000. Dislocation patterning: from micro- to mesoscale description. Phys. Rev. Lett. 84, $1487-1490$.

Groma, I., Pawley, G.S., 1993a. Computer simulation of plastic behavior of single crystals. Philos. Mag. A 67, $1459-1470$.

Groma, I., Pawley, G.S., 1993b. Role of secondary slip system in a computer simulation model of plastic behavior of single crystals. Mater. Sci. Eng. A 164, 306-311.

Gullouglu, A.N., Hartly, C.S., 1993. Simulation of dislocation microstructures in two dimensions. II. Dynamic and relaxed structures. Modelling Simul. Mater. Sci. Eng. 1 (4), 383-402.

Gullouglu, A., Srolovitz, D., LeSar, R., Lomdahl, P., 1989. Dislocation distributions in two dimensions. Scr. Metall. 23 , $1347-1352$.

Hähner, P., 1996. On the foundations of stochastic dislocation dynamics. Appl. Phys. A 62, 473-481.

Hirth, J.P., Lothe, J., 1992. Theory of Dislocations. Reprint ed. Krieger, New York, p. 105 (chapter 4).

Holt, D.L., 1970. Dislocation cell formation in metals. J. Appl. Phys. 41, 3197-3201.

Kosevich, A.M., 1962. The deformation of field in an isotropic elastic medium containing moving dislocations. Sov. Phys. JETP 15, 108-115.

Koslowski, M., Ortiz, M., 2004. A multi-phase field model of planar dislocation networks. Modelling Simul. Mater. Sci. Eng. 12, 1087-1097.

Kratochvil, J., 1990a. Derivation of mughrabi's cellular structure model from synergetics of dislocations. Scr. Metall. Mater. $24,891$.

Kratochvil, J., 1990b. Instability origin of dislocation cell misorientation. Scr. Metall. Mater. 24, 1225-1228.

Kröner, E., 1958. Kintinuumstheorie der Versetzungen und Eigenspannungen. Springer, Berlin.

Kuhlmann-Wilsdorf, D., 1989. Theory of plastic deformation: properties of low energy dislocation structures. Mater. Sci. Eng. A 113, $1-41$.

Kuhlmann-Wilsdorf, D., 1995. Technological high strain deformation of 'wavy glide' metals and leds. Phys. Status Solidi (a) 149, $225-241$. Langer, J.S., 1998. Microstructural shear localization in plastic deformation of amorphous solids. Phys. Rev. E 64, $011504-011506$.

Lepinoux, J., Kubin, L.P., 1987. The dynamic organization of dislocation structures: a simulation. Scr. Metall. 21, 833-838.

Limkumnerd, S., Sethna, J.P., 2006. Mesoscale theory of grains and cells: crystal plasticity and coarsening. Phys. Rev. Lett. $96,095503$. 
Limkumnerd, S., Sethna, J.P., 2007. Stress-free states of continuum dislocation fields: rotations, grain boundaries, and the Nye dislocation density tensor. Phys. Rev. B 75, 224121.

Lubarda, V.A., Blume, J.A., Needleman, A., 1993. An analysis of equilibrium dislocation distributions. Acta Metall. Mater. 41, 625-642.

Ma, A., Roters, F., Raabe, D., 2006. A dislocation density based constitutive model for crystal plasticity fem including geometrically necessary dislocations. Acta Materialia 54, 2169-2179.

Miguel, M.C., Vespignani, A., Zapperi, S., Weiss, J., Grasso, J.R., 2001. Complexity in dislocation dynamics: model. Mater. Sci. Eng. A 309-310, 324-327.

Mika, D.P., Dawson, P.R., 1999. Polycrystal plasticity modelling of intracrystalline boundary textures. Acta. Mater. 47, $1355-1369$.

Mura, T., 1963. Continuous distribution of moving dislocations. Philos. Mag. 8, 843-857.

Nye, J.F., 1953. Some geometrical relations in dislocated crystals. Act. Metall. 1, 153-162.

Ortiz, M., Repetto, E.A., 1999. Nonconvex energy minimization and dislocation structures in ductile single crystals. J. Mech. Phys. Solids 47, 397-462.

Ortiz, M., Repetto, E.A., Stainier, L., 2000. A theory of subgrain dislocation structures. J. Mech. Phys. Solids 48, $2077-2114$.

Pontes, J., Walgraef, D., Aifantis, E.C., 2005. On dislocation patterning: multiple slip effects in the rate equation approach. Int. J. Plast. 22, 1486-1505.

Raabe, D., Becker, R.C., 2000. Coupling of a crystal plasticity finite-element model with a probabilistic cellular automaton for simulating primary static recrystallization in aluminium. Modeling Simul. Mater. Sci. Eng. 8, 445-462.

Ramasubramaniam, A., Ariza, M.P., Ortiz, M., 2007. A discrete mechanics approach to dislocation dynamics in bcc crystals. J. Mech. Phys. Solids 55, 615-647.

Rickman, J.M., Vinãls, 1997. Modelling of dislocation structures in materials. Philos. Mag. A 75 (5), 1251-1262.

Roy, A., Acharya, A., 2005. Finite element approximation of field dislocation mechanics. J. Mech. Phys. Solids 53, $143-170$.

Saxlová, M., Kratochvil, J., Zatloukal, J., 1997. The model of formation and disintegration of vein dislocation structure. Mater. Sci. Eng. A $234-236,205-208$.

Sethna, J.P., Rauscher, M., Bouchaud, J.-P., 2004. Continuum mesoscale theory inspired by plasticity. Europhysics Lett. $65,665$.

Walgraef, D., Aifantis, E.C., 1985. On the formation and stability of dislocation patterns-I, -II, -III. Int. J. Eng. Sci. 23 , $1351-1372$.

Whitham, G.B., 1974. Linear and Nonlinear Waves. Wiley-Interscience, New York.

Zaiser, M., 2006. Scale-invariance in plastic flow of crystalline solids. Adv. Phys. 55, 85-245.

Zaiser, M., Miguel, M.C., Groma, I., 2001. Statistical dynamics of dislocation systems: the influence of dislocation-dislocation correlations. Phys. Rev. B 64, 224102. 